\title{
Análise do equilíbrio corporal e aptidão física de indivíduos em tratamento para dependência química
}

\section{Analysis of body balance and physical fitness of individuals under treatment for chemical dependence}

\section{AUTORES \\ Bruno Marson Malagodi ${ }^{1}$ (D) \\ Márcia Greguol2 (D) \\ Hélio Serassuelo Junior ${ }^{2}$ (D) \\ 1 Programa de Pós-Graduacão em Educacão Física

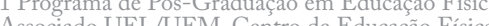 e Esporte, Universidade Estadual de Londrina Universidade Estadual de Maringá, Londrina, Paraná, Brasil. \\ 2 Departamento de Ciências do Esporte, Centro de Educação Física e Esporte, Universidade Estadual de Londrina, Londrina, Paraná, Brasil.}

\section{CONTATO}

Bruno Marson Malagodi

brunomarson32@gmail.com

Centro de Educação Física e Esporte, Universidade Estadual de Londrina, Rodovia Celso Garcia Cid, Pr 445 Km 380, Campus Universitário, Londrina, Paraná, Brasil. CEP 86057-970.

DOI

10.12820/rbafs.23e0012

\begin{abstract}
RESUMO
O abuso de álcool e drogas pode ocasionar efeitos negativos nas habilidades motoras e capacidades coordenativas. Diversos estudos têm demonstrado a precariedade nos níveis de aptidão física em indivíduos dependentes químicos, sendo que variáveis da aptidão física e equilíbrio corporal são influenciados pelo consumo prolongado dessas substâncias mesmo após a cessação do uso. O objetivo do presente estudo foi avaliar indicadores da aptidão física e equilíbrio corporal de indivíduos internados para tratamento de dependência química, relacionando os dados ao tempo de uso de drogas ou álcool. Foram analisados indicadores antropométricos, flexibilidade, agilidade e equilíbrio de 46 indivíduos adultos (14 mulheres e 32 homens com 38,3 $\pm 12,9$ anos), que também responderam uma anamnese. Utilizou-se estatística descritiva, teste t student, Mann Whitney e correlação de Spearman, adotando-se $\mathrm{p} \leq 0,05$. Os resultados obtidos demonstram maior prevalência de pacientes do sexo masculino, dependentes de múltiplas substâncias, baixo nível de escolaridade, tempo de consumo superior a 10 anos e que não praticavam atividade física regular previamente à internação. Quanto maior o tempo de uso, piores são os resultados nos testes de agilidade e equilíbrio, tanto com olhos abertos quanto com os olhos fechados. É possível concluir que tanto o avançar da idade quanto o maior tempo de dependência pioram o desempenho nas variáveis agilidade e equilíbrio corporal. Dessa forma, ressalta-se a importância em oferecer programas de atividade física que aprimorem aspectos da aptidão física relacionada à saúde de indivíduos internados para tratamento de dependência química, sobretudo para aqueles com maior tempo de uso de drogas e álcool.
\end{abstract}

Palavras-chave: Alcoolismo; Abuso de drogas; Equilíbrio postural; Aptidão física.

ABSTRACT

Alcohol and drugs abuse can have negative effects on motor skills and physical capabilities. Several studies have demonstrated the precariousness of the physical fitness levels of individuals with chemical dependence, and that variables of physical fitness and body balance are infuenced by prolonged consumption of these substances even after cessation of use. The objective of the study was to evaluate indicators of physical fitness and body balance of individuals hospitalized for treatment of chemical dependence, relating the data to the time of drug or alcohol use. Anthropometric, flexibility, agility and balance indicators of 46 adult individuals (14 women and 32 men with $38.3 \pm 12.9$ years) were analyzed, and they also answered an anamnesis. Descriptive statistics, Student's test, Mann Whitney and Spearman's correlation were used, adopting $p \leq 0.05$. Results showed a higher prevalence of male patients, dependent on multiple substances, low level of schooling, consumption time of more than 10 years and who did not practice regular physical activity prior to hospitalization. The longer the time of drug use, the worse the results in the agility and balance tests, both with open eyes and closed eyes. It is possible to conclude that both the advancing age and the greater dependence time worsen performance in the variables agility and body balance Therefore, it is important to offer programs of physical activity that improve aspects of physical fitness related to the health of individuals hospitalized for treatment of chemical dependence, especially for those with a longer time of drug and alcohol use.

Keywords: Alcoholism; Drug abuse; Postural balance; Physical fitness.

\section{Introdução}

A dependência química é um transtorno mental caracterizado por um grupo de sinais e sintomas decorrentes do uso de drogas ${ }^{1}$. Dentre estes, destacam-se a compulsão e a falta de controle no uso da droga, sintomas de abstinência, necessidade de doses crescentes para atin- gir o mesmo efeito, abandono de outras atividades e manutenção do uso, mesmo tendo prejuízos evidentes ${ }^{1}$.

Cada droga de abuso tem o seu mecanismo de ação particular, mas todas atuam direta ou indiretamente ativando uma mesma região do cérebro: o sistema de recompensa cerebral, formado por circuitos neuronais 
responsáveis pelas ações de reforço positivo e negativo $^{2}$. Uma característica importante dos transtornos por uso de substâncias é uma alteração básica nos circuitos cerebrais que pode persistir mesmo após a desintoxicação, especialmente em indivíduos com transtornos graves. Os efeitos comportamentais dessas alterações cerebrais podem ser exibidos nas recaídas constantes e na fissura intensa por drogas quando os indivíduos são expostos a estímulos relacionados a elas ${ }^{1}$.

Segundo Stenbacka, Leifman e Romelsjö ${ }^{3}$ além dos transtornos mentais e comportamentais, os dependentes químicos apresentam maior prevalência de doenças crônicas, tais como doença cardiovascular, câncer, além de uma expectativa de vida entre 15 e 20 anos mais baixa que a população em geral, sendo a menor entre pessoas com doenças mentais. Vários fatores contribuem para os problemas mentais e físicos que acometem esses indivíduos, como maior tendência ao comportamento sedentário, alimentação inadequada, além da exposição precoce e repetida às propriedades químicas da substância utilizada ${ }^{3}$.

A melhoria da aptidão física de pacientes em tratamento para dependência química pode ser um importante fator que contribuirá para prevenção das comorbidades físicas associadas ao estado de saúde geral desses indivíduos ${ }^{4}$. Alguns estudos têm demonstrado a precariedade dos níveis de aptidão física de indivíduos com dependência química, encontrando menores índices de aptidão cardiorrespiratória e força muscular em indivíduos dependentes químicos quando comparados à população em geral e, consequentemente, maior risco de desenvolver doenças cardiovasculares, morte prematura e outras doenças relacionadas ao estilo de vida ${ }^{5,6}$.

Schmidt e colaboradores ${ }^{7}$ afirmam que o consumo a longo prazo de drogas lícitas e ilícitas podem ocasionar efeitos colaterais crônicos que influenciam negativamente as habilidades motoras e capacidades coordenativas, como o tempo de reação, a coordenação óculo-manual e o equilíbrio, mesmo após a cessação do uso. As alterações na função do aparelho vestibular e do cerebelo, em virtude do consumo dessas substâncias, poderiam ocasionar sintomas como vertigem, tontura e perda de equilíbrio, prejudicando a autonomia e qualidade de vida ${ }^{8}$.

Quanto ao tempo de consumo de drogas e álcool ao longo da vida por indivíduos em situação de dependência química, algumas pesquisas têm reportado que esta variável, juntamente com a quantidade total da substância consumida, possui relação direta com a degene- ração cerebelar ${ }^{9}$, sendo que o maior tempo de exposição prejudicaria de maneira progressiva a manutenção da postura e do equilíbrio corporal ${ }^{10}$. No entanto, não foram localizados estudos que analisassem esta relação envolvendo outras variáveis da aptidão física em indivíduos nesta condição.

Devido à escassez de estudos na área e tendo em vista a relevância do tema, o presente estudo teve como objetivo analisar o equilíbrio corporal e variáveis da aptidão física em indivíduos internados para tratamento de dependência química, relacionando-as com o tempo de uso de drogas e álcool pelos participantes.

\section{Método}

O estudo realizado foi descritivo correlacional, com delineamento transversal. Os dados foram coletados na instituição CREDEQUIA (Centro de Recuperação de Dependentes Químicos e Alcoólatras) localizada na cidade de Londrina/Paraná, que atende indivíduos de ambos os sexos e oferece a prática da atividade física como parte do processo terapêutico. A seleção da amostra ocorreu por conveniência, sendo que todos os indivíduos que se encaixavam nos critérios de inclusão foram convidados e aceitaram participar do estudo. Um tamanho amostral de 42 indivíduos foi estimado pelo software $G^{*}$ Power 3.1 .9 para um poder $(1-\beta)$ de $80 \%$, nível de significância unicaudal $(\alpha)$ de 0,05 e tamanho de efeito elevado $(d=0,8)$. Ao final, a amostra foi constituída por 46 indivíduos adultos (sendo 14 mulheres e 32 homens) com idade média de 38,3 $\pm 12,9$ anos.

Para participar do estudo, o indivíduo deveria ser maior de 18 anos de idade e estar iniciando tratamento voluntário para dependência química na comunidade terapêutica. Foram inclusos apenas os indivíduos que estavam na fase inicial do tratamento. Para que fossem evitadas possíveis influências dos sintomas agudos da abstinência do uso de substâncias psicoativas na realização dos testes, os indivíduos foram avaliados apenas no início da terceira semana de internação. Porém, caso algum paciente apresentasse ainda neste período sintomas agudos da síndrome de abstinência relatados pelo médico da instituição, tais como taquicardia, sudorese e tremores excessivos, ânsia de vômito, fissura para o uso da substância, entre outros, o mesmo seria excluído da pesquisa. Antes da aplicação dos testes, todos os pacientes passaram por consultas iniciais com o médico da Comunidade Terapêutica, o qual analisou os resultados de exames clínicos (avaliação do comportamento da pressão arterial e do eletrocardiograma em situa- 
ção de repouso), a condição de marcha independente e a função cognitiva (Mini Exame do Estado Mental - MEEM) ${ }^{11}$. Foram considerados aptos para a participação na pesquisa os indivíduos que apresentassem marcha independente, comportamentos pressórico e eletrocardiográfico normais e condição cognitiva mínima para a compreensão dos instrumentos de pesquisa (MEEM $\geq 21$ pontos). Todos os indivíduos foram classificados como portadores da síndrome da dependência de substâncias psicoativas, de acordo com os critérios da Classificação Internacional de Doenças (CID-10 ${ }^{12}$.

Todos os participantes assinaram um Termo de Consentimento Livre e Esclarecido (TCLE) concordando em participar da pesquisa e apresentaram liberação médica para a prática de exercícios físicos. $\mathrm{O}$ estudo foi aprovado pelo Comitê de Ética em Pesquisa com Seres Humanos da Universidade Estadual de Londrina (Número do Parecer: 2.125.747/2017).

No primeiro dia de avaliação os indivíduos foram informados sobre a natureza da pesquisa, assinaram o TCLE e responderam uma anamnese previamente elaborada, com informações sobre idade, escolaridade, tipo de substância consumida, tempo de uso/abuso, uso de medicamentos e outras condições de saúde. Ainda nesta anamnese, aplicada na forma de entrevista individual com cada participante, era questionado se o mesmo praticava ou não alguma atividade física regular no mês precedente à internação. Caso o indivíduo relatasse que praticava alguma atividade de maneira regular no período, era questionado o tipo de atividade, número de sessões semanais e duração das mesmas).

Posteriormente, foram realizadas medidas das variáveis antropométricas: massa corporal (por meio de balança digital com precisão de 100 gramas), estatura (por meio de estadiômetro com precisão de 0,1 centímetro) e perímetro de cintura (com trena antropométrica de dois metros). A partir desses valores, foi calculado o IMC (índice de massa corporal) e a RCE (relação cintura/estatura) de todos os indivíduos. A classificação do IMC obedeceu aos critérios propostos pela Organização Mundial da Saúde, (eutrofia se IMC $<25 \mathrm{~kg} / \mathrm{m}^{2}$, sobrepeso se IMC entre 25 e $29,9 \mathrm{~kg} / \mathrm{m}^{2}$ e obesidade para IMC $\geq 30 \mathrm{~kg} / \mathrm{m})^{13}$.

No segundo dia os indivíduos realizaram testes de variáveis da aptidão física:

Teste de equilíbrio em posição semiestática com apoio bipodal utilizando a plataforma de pressão plantar da marca myoPressure Noraxon ${ }^{\circledR}$. Os indivíduos permaneciam na posição bipodal por 30 segundos em duas situações distintas - olhos abertos e fechados. Os dados registrados foram armazenados e posteriormente analisados pelo software específico Noraxon ${ }^{\circledR}$ myoRESEARCH 3.10. Para avaliar o equilíbrio dos indivíduos foi considerado o comportamento do COP Path Length [a distância percorrida pelo COP (Centro de Pressão) em milímetros $(\mathrm{mm})$ durante o tempo de análise], o COP Vel (variação do deslocamento do centro de pressão através do tempo em $\mathrm{mm} / \mathrm{s}$ ) e a área da Elipse (quantifica 95\% da área total percorrida pelo centro de pressão nos eixos médio lateral e ântero posterior usando uma elipse para ajustar os dados), sendo que, quanto menores os valores registrados nas variáveis, melhor o equilíbrio analisado ${ }^{14}$.

Análise da flexibilidade na região posterior do tronco, articulação do quadril e membros inferiores foi realizando o Teste de Sentar e Alcançar (TSA) em banco específico, proposto originalmente por Wells \& Dil$\operatorname{lon}^{15}$. Cada indivíduo realizou três tentativas sucessivas e foi considerada apenas a melhor marca obtida (em centímetros).

Análise da agilidade, ou seja, a capacidade de mudar rápida e eficientemente a direção em velocidade, por meio do teste $\mathrm{T}$ (Figura 1) proposto por Pauole ${ }^{16} \mathrm{O}$ percurso total do teste foi de 40 metros percorridos em formato de $\mathrm{T}$, sendo considerado o menor tempo de duas tentativas realizadas (em segundos). Ao ser dado o comando verbal para o teste, o indivíduo iniciava a corrida e, ao atingir a linha de partida, o cronômetro era acionado, com o objetivo de medir o tempo gasto até a finalização do percurso de 40 metros, quando então o cronômetro era travado.

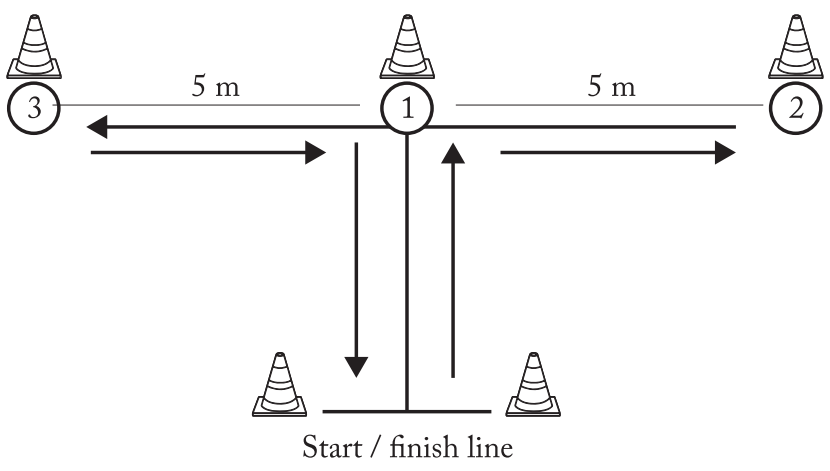

Figura 1 - Teste $\mathrm{T}$ de agilidade ${ }^{16}$.

A normalidade dos dados foi analisada por meio do teste de Shapiro Wilk. A partir daí, os dados foram inicialmente tratados de modo descritivo. Em seguida, para determinar as diferenças das variáveis da aptidão 
física analisadas por sexo, grupo etário (até 40 anos e mais de 40 anos), tempo de dependência química (até 10 anos e mais de 10 anos, por conta dos efeitos mais pronunciados que a dependência química pode trazer após este período ${ }^{17}$ ) e prática de atividade física (se praticava antes da internação ou não), foram utilizados teste t-student para amostras independentes e o teste U de Mann-Whitney. Já as comparações entre os diferentes tipos de drogas utilizadas foram feitas através de Análise de Variância (ANOVA), com post hoc de Scheffé. Por fim, as relações entre as variáveis foram verificadas por meio de teste de Correlação de Spearman. Em todas as situações, a significância adotada foi $\mathrm{p} \leq 0,05$.

\section{Resultados}

Inicialmente são apresentados os dados descritivos dos participantes do estudo na Tabela 1. Em seguida, são discriminadas as análises comparativas e correlacionais dos indicadores da aptidão física, equilíbrio corporal e demais variáveis intervenientes.

Tabela 1 - Valores de média e desvio padrão das variáveis do estudo ( $\mathrm{n}=46$, Londrina, 2018).

\begin{tabular}{lcc}
\hline Variável & Média & Desvio Padrão \\
\hline Idade (anos) & 38,33 & 12,92 \\
Tempo de uso de drogas (anos) & 19,13 & 13,91 \\
Estatura (cm) & 167,65 & 8,65 \\
Massa (kg) & 70,29 & 11,23 \\
IMC (kg/m²) & 24,95 & 3,22 \\
Cintura (cm) & 90,01 & 9,47 \\
RCE & 0,54 & 0,061 \\
Agilidade (segundos) & 20,81 & 8,14 \\
Flexibilidade (cm) & 24,35 & 7,86 \\
COP_Path_OA (mm) & 219,19 & 121,91 \\
COP_Path_OF (mm) & 372,22 & 243,24 \\
COP_Vel_OA (mm/s) & 7,65 & 5,38 \\
COP_Vel_OF $(\mathrm{mm} / \mathrm{s})$ & 13,78 & 14,04 \\
Elipse_OA $\left(\mathrm{mm}{ }^{2}\right)$ & 182,61 & 126,61 \\
Elipse_OF $\left(\mathrm{mm}{ }^{2}\right)$ & 308,46 & 253,94 \\
\hline
\end{tabular}

RCE: Relação cintura/estatura; OA: olhos abertos; OF: olhos fechados; $\mathrm{COP}=$ centro de pressão.

Foi possível observar maior prevalência de homens (69,6\%), com dependência múltipla, ou seja, dependência em mais de uma substância (54,3\%, contra 34,8\% usuários de álcool e 10,9\% usários de cocaínal crack). A maioria dos indivíduos $(60,9 \%)$ relatou utilizar drogas por mais de 10 de anos. Em geral observa-se baixo nível de escolaridade, com 67,4\% dos indivíduos relatando ter cursado nível fundamental incompleto ou completo e apenas 8,7\% dos indivíduos com o ensino superior completo. As idades dos participantes variaram dos 19 aos 56 anos.

A prática de atividade física regular antes da internação de pelo menos 150 minutos por semana foi relatada por apenas 16 indivíduos $(34,8 \%)$, sendo 12 homens (37,5\% dos homens) e quatro mulheres (28,6\% das mulheres). As atividades relatadas foram: caminhadas três a quatro vezes por semana com $45 \mathrm{a}$ 60 minutos por dia (9 casos); natação duas vezes por semana mais caminhada uma vez por semana, sempre com 60 minutos ao dia (3 casos); treinamento com pesos três vezes por semana com 60 a 90 minutos por dia (3 casos); futebol uma vez por semana e treinamento com pesos duas vezes por semana, com 60 minutos ao dia (1 caso). O uso de medicação psicotrópica para tratamento de comorbidades e coadjuvantes no processo terapêutico foi relatado por $52,2 \%$. Para a relação do IMC, apenas dois indivíduos foram considerados obesos (duas mulheres), 19 foram considerados com sobrepeso $(41,3 \%)$ e os demais 25 indivíduos $(54,3 \%)$ foram considerados eutróficos. Quando comparados os diferentes níveis de escolaridade, não houve diferença significativa em nenhuma das variáveis da aptidão física pesquisadas. Quando comparadas as variáveis por sexo dos indivíduos, a única diferença observada foi na estatura, sendo os homens significativamente mais altos do que as mulheres $(171,0+5,9 \mathrm{~cm}$ para os homens contra $159,9+9,0)$.

Em relação aos grupos que faziam e que não faziam atividade física previamente à internação, verificou-se que aqueles mais ativos fisicamente obtiveram melhores resultados no teste de flexibilidade utilizando o banco de Wells $(27,8+6,6 \mathrm{~cm}$ para os ativos contra 22,5+7,9 cm para os sedentários, com $\mathrm{p}=0,027$ ). Não houve diferença significativa do tempo de uso de substâncias entre aqueles que praticavam ou não praticavam atividade física. Já as comparações por tempo de dependência, tipo de substância utilizada e grupo etário (até 40 anos e mais de 40 anos) encontram-se detalhadas na Tabela 2.

Comparando os grupos com até 10 anos de uso e com mais de 10 anos de uso, foi visto que o tempo no teste de agilidade foi maior nos indivíduos que consumiam drogas há mais tempo, indicando pior resultado nesta variável $(p=0,010)$. O equilíbrio (COP PATH, COP VEL e área da Elipse) foi pior também para os usuários de mais de 10 anos, tanto na situação de olhos abertos $(p=0,001)$ 
Tabela 2 - Comparações das variáveis por tempo de dependência, tipo de substância consumida e grupo etário.

\begin{tabular}{|c|c|c|c|c|c|c|c|}
\hline \multirow[b]{2}{*}{ VARIÁVEL } & \multicolumn{2}{|c|}{ Tempo de uso } & \multicolumn{3}{|c|}{ Substância utilizada } & \multicolumn{2}{|c|}{ Idade } \\
\hline & $\begin{array}{l}\text { Até } 10 \text { anos } \\
(\mathrm{n}=18)\end{array}$ & $\begin{array}{l}\text { Mais de } 10 \text { anos } \\
\quad(\mathrm{n}=28)\end{array}$ & $\begin{array}{l}\text { Álcool } \\
(\mathrm{n}=16)\end{array}$ & $\begin{array}{c}\text { Cocaína/crack } \\
(\mathrm{n}=5)\end{array}$ & $\begin{array}{l}\text { Múltiplas } \\
(\mathrm{n}=25)\end{array}$ & $\begin{array}{l}\text { Até } 40 \text { anos } \\
(\mathrm{n}=20)\end{array}$ & $\begin{array}{c}\text { Mais de } 40 \text { anos } \\
\quad(\mathrm{n}=26)\end{array}$ \\
\hline Idade (anos) & $27,8 \pm 8,8$ & $45,1 \pm 10,4^{*}$ & $48,8 \pm 11,6^{*}$ & $35,4 \pm 7,6$ & $32,2 \pm 10,3$ & $29,5 \pm 7,9$ & $49,9 \pm 8,1$ \\
\hline Uso (anos) & $5,3 \pm 3,1$ & $28,0 \pm 10,3^{*}$ & $30,0 \pm 13,9^{*}$ & $9,2 \pm 8,9$ & $14,2 \pm 10,1$ & $11,8 \pm 7,9$ & $28,7 \pm 14,3 *$ \\
\hline Estatura $(\mathrm{cm})$ & $167,3 \pm 11,8$ & $167,9 \pm 6,1$ & $167,7 \pm 6,9$ & $160,0 \pm 10,0$ & $169,2 \pm 8,9$ & $170,9 \pm 7,1$ & $163,4 \pm 8,7^{*}$ \\
\hline Massa $(\mathrm{kg})$ & $70,6 \pm 15,0$ & $70,1 \pm 8,3$ & $71,3 \pm 7,5$ & $71,9 \pm 18,3$ & $69,3 \pm 11,9$ & $73,5 \pm 11,5$ & $66,1 \pm 9,6$ \\
\hline $\operatorname{IMC}\left(\mathrm{kg} / \mathrm{m}^{2}\right)$ & $25,1 \pm 4,2$ & $24,9 \pm 2,5$ & $25,4 \pm 2,6$ & $27,8 \pm 5,5$ & $24,1 \pm 2,7$ & $25,1 \pm 3,7$ & $24,7 \pm 2,5$ \\
\hline Cintura $(\mathrm{cm})$ & $88,9 \pm 11,7$ & $90,7 \pm 7,9$ & $95,1 \pm 7,6$ & $94,2 \pm 15,0$ & $85,9 \pm 7,5^{*}$ & $88,9 \pm 10,0$ & $91,5 \pm 8,7^{*}$ \\
\hline $\mathrm{RCE}$ & $0,5 \pm 0,1$ & $0,5 \pm 0,1$ & $0,6 \pm 0,06$ & $0,6 \pm 0,09$ & $0,5 \pm 0,1^{*}$ & $0,5 \pm 0,1$ & $0,6 \pm 0,1 *$ \\
\hline Agilidade (seg) & $17,0 \pm 4,8$ & $23,3 \pm 8,9^{*}$ & $24,3 \pm 8,1$ & $19,4 \pm 5,6$ & $18,9 \pm 8,1$ & $18,1 \pm 7,7$ & $24,3 \pm 7,5 *$ \\
\hline Flexibilidade $(\mathrm{cm})$ & $25,9 \pm 7,2$ & $23,3 \pm 8,2$ & $23,9 \pm 8,2$ & $24,2 \pm 7,9$ & $24,7 \pm 7,9$ & $24,7 \pm 7,6$ & $23,9 \pm 8,3$ \\
\hline COP_Path_OA (mm) & $157,8 \pm 58,1$ & $258,6 \pm 136,1^{*}$ & $264,0 \pm 153,2$ & $180,2 \pm 63,8$ & $198,3 \pm 102,1$ & $215,3 \pm 117,8$ & $224,3 \pm 129,9$ \\
\hline COP_Path_OF (mm) & $266,3 \pm 108,1$ & $440,3 \pm 281,0^{*}$ & $463,4 \pm 319,4$ & $307,0 \pm 102,2$ & $326,9 \pm 191,9$ & $356,8 \pm 203,5$ & $392,3 \pm 291,4$ \\
\hline COP_Vel_OA $(\mathrm{mm} / \mathrm{s})$ & $5,3 \pm 1,9$ & $9,1 \pm 6,3^{*}$ & $9,7 \pm 7,7$ & $6,2 \pm 2,2$ & $6,6 \pm 3,4$ & $7,81 \pm 6,2$ & $7,5 \pm 4,3$ \\
\hline COP_Vel_OF $(\mathrm{mm} / \mathrm{s})$ & $8,8 \pm 3,5$ & $16,9 \pm 17,2^{*}$ & $5,4 \pm 10,6$ & $10,2 \pm 3,3$ & $13,4 \pm 17,1$ & $14,4 \pm 16,4$ & $13,0 \pm 9,6$ \\
\hline Elipse_OA $\left(\mathrm{mm}^{2}\right)$ & $134,9 \pm 78,9$ & $213,3 \pm 142,9^{*}$ & $210,1 \pm 148,1$ & $167,4 \pm 94,9$ & $168,1 \pm 119,2$ & $190,5 \pm 131,4$ & $172,4 \pm 123,3$ \\
\hline Elipse_OF $\left(\mathrm{mm}^{2}\right)$ & $201,4 \pm 150,3$ & $377,3 \pm 284,1^{*}$ & $306,1 \pm 219,8$ & $255,4 \pm 220,2$ & $320,6 \pm 285,4$ & $311,6 \pm 283,9$ & $304,4 \pm 215,9$ \\
\hline
\end{tabular}

$\mathrm{RCE}=$ Relação cintura/estatura; $\mathrm{IMC}=$ Índice de Massa Corporal; $\mathrm{AO}=$ olhos abertos; $\mathrm{OF}$ = olhos fechados.; COP Path AO = distância percorrida pelo centro de pressão com olhos abertos; COP Path OF = distância percorrida pelo centro de pressão com olhos fechados; COP $\mathrm{Vel} \mathrm{AO}$ = variação do deslocamento do centro de pressão através do tempo com olhos abertos; COP Vel OF = variação do deslocamento do centro de pressão através do tempo com olhos fechados. * $p<0,05$.

quanto na situação de olhos fechados $(\mathrm{p}=0,005)$.

Sobre a idade média dos indivíduos, quando comparados pelo tipo de droga que utilizavam, verificou-se que os dependentes de álcool eram significativamente mais velhos $(p=0,000)$ e utilizavam a droga a um tempo significativamente maior $(p=0,000)$. Ainda comparando o tipo de droga, a medida de cintura dos dependentes múltiplos foi significativamente menor ( $\mathrm{p}$ = 0,004). Da mesma forma, a RCE foi menor para o grupo de dependentes múltiplos $(p=0,001)$.

Os sujeitos foram divididos em dois grupos etários para a análise: até 40 anos e acima de 40 anos. Comparando os grupos, o grupo acima de 40 anos obteve o pior desempenho no teste de agilidade, exibindo um tempo maior $(p=0,009)$. Da mesma forma, exibiram maior $\operatorname{RCE}(p=0,027)$ e menor estatura $(p=0,002)$.

Foi realizado ainda o teste de correlação de Spearman para verificar as relações significativas entre as variáveis de estudo considerando o grupo como um todo. Observou-se que a idade mostrou correlação positiva com o tempo no teste de agilidade $(\mathrm{R}=0,63$; $\mathrm{p}=0,000)$, com o COP PATH na situação de olhos abertos $(R=0,37 ; p=0,003)$ e fechados $(R=0,43 ; p$ $=0,003)$ e com a área da Elipse de olhos fechados ( $R$ $=0,31 ; \mathrm{p}=0,039)$. Isso pode demonstrar que, quanto maior o avanço etário, maior o tempo no teste de agilidade (pior resultado para a agilidade) e pior o resultado em alguns parâmetros do equilíbrio, especialmente na situação de olhos fechados.

Já o tempo em anos em que o indivíduo usou drogas ou álcool teve relação positiva com o tempo no teste de agilidade $(\mathrm{R}=0,55 ; \mathrm{p}=0,000)$ e com todas as variáveis do equilíbrio (COP PATH, COP VEL e Área da Elipse) na situação de olhos abertos $(\mathrm{R}=0,48 ; \mathrm{p}=0,001 ; \mathrm{R}$ $=0,37 ; \mathrm{p}=0,011 ; \mathrm{R}=0,33 ; \mathrm{p}=0,027$, respectivamente), mostrando que, quanto maior o tempo de uso de drogas, piores são os resultados no teste de agilidade e de equilíbrio, tanto com olhos abertos quanto com os olhos fechados.

\section{Discussão}

O objetivo do estudo foi analisar o equilíbrio corporal e indicadores da aptidão física de indivíduos internados voluntariamente para o tratamento de dependência química em uma comunidade terapêutica.

$\mathrm{Na}$ análise descritiva dos participantes do estudo, observou-se entre os pacientes internados maior prevalência de pacientes do sexo masculino, dependentes de múltiplas substâncias. Segundo dados da Secretaria Nacional de Políticas sobre Drogas ${ }^{18}$ o uso, abuso e dependência de álcool e outras drogas são mais frequentes em homens, embora essa diferença venha diminuindo ao longo dos anos especialmente nas faixas etárias mais jovens. Como exemplo é citado que a dependência de 
álcool entre adultos no Brasil é de 19,5\% entre os homens e $6,9 \%$ entre as mulheres. Já entre os adolescentes entre 12 e 17 anos, a prevalência é 7,3\% para os meninos e 6,0\% para as meninas, ou seja, a ocorrência é muito semelhante em ambos os sexos. No entanto, no presente estudo foram avaliados apenas indivíduos adultos, e tal fato pode explicar a maior prevalência de homens participantes.

Outro dado observado foi a maior porcentagem de indivíduos com baixa escolaridade e com tempo de dependência superior a 10 anos. Segundo a Organização Mundial da Saúde ${ }^{19}$, o início do consumo de álcool, cigarro e outras drogas ocorrem predominantemente durante a adolescência, o que pode justificar o fato de a maior parte dos indivíduos consumirem o álcool e outras substâncias por um logo período antes de buscarem tratamento. Ainda, como em muitos casos, o início à dependência é precoce, o que geralmente acarreta $o$ abandono escolar em idades mais jovens, o que explicaria a baixa escolaridade observada.

Com relação aos dados do IMC, a maioria dos indivíduos foi classificada dentro dos limites da eutrofia. Esses dados corroboram os achados de Willhelm, Escobar e Perry ${ }^{20}$, que detectaram maior porcentagem de indivíduos eutróficos entre dependentes de crack internados em uma unidade de tratamento de dependência química. Ainda segundo os autores, deve-se atentar para a possibilidade de aumento acentuado da massa corporal desses indivíduos ao longo do tratamento, uma vez que os indivíduos em geral chegam à internação muito debilitados e, por conta da abstinência, podem desenvolver um mecanismo de hiperfagia rebote. Esse mecanismo funcionaria para restabelecer os neurotransmissores (dopamina e serotonina, principalmente) do sistema de recompensa, o que pode gerar abrupto aumento da massa corporal e consequente estado de sobrepeso e obesidade.

Outro ponto observado foi a baixa prevalência de indivíduos que praticavam atividade física regular previamente à internação. No entanto, estes dados devem ser interpretados com cautela, visto que algumas sequelas da dependência química crônica, tais como a fraqueza, problemas de coordenação motora e equilíbrio, podem reduzir a participação em programas de atividades físicas. Dentre as várias consequências do comportamento sedentário, é possível destacar entre usuários de álcool e outras drogas elevado grau de isolamento social, qualidade de vida precária, além de baixa autoestima ${ }^{21}$. Ain$\mathrm{da}$, conforme observado no presente estudo, a falta de atividade física regular impacta negativamente a aptidão física relacionada à saúde, visto que os indivíduos eram ativos previamente à internação exibiram melhores resultados nos testes de flexibilidade e agilidade quando comparados àqueles que não faziam atividade física.

Entre homens e mulheres, melhoras relativas na aptidão física e na prática de atividade física habitual são associadas à menor sintomatologia depressiva e maior bem estar emocional ${ }^{22}$. Tais associações são consistentes com a convicção de que a atividade física é um comportamento particularmente benéfico para pessoas com doenças crônicas, incluindo transtorno depressivo grave e doenças cardiovasculares, devido aos efeitos combinados tanto em termos físicos como na saúde mental ${ }^{23}$. Cabe destacar que os danos provocados pelos efeitos psicoativos das drogas podem afetar cronicamente o funcionamento mental, tanto pela dependência desencadeada pelo uso contínuo ou pelos efeitos tóxicos da droga no corpo.

Ao se comparar os usuários pelo tipo de droga consumida, verificou-se que aqueles com dependência de álcool eram significativamente mais velhos e com maior tempo de dependência. Isso pode significar que os dependentes de álcool demoram mais para buscar o tratamento, visto que o álcool é uma droga considerada "aceitável" e lícita na sociedade. Os resultados do presente estudo também mostraram que os pacientes com maior tempo de dependência exibiram piores resultados nos testes de agilidade (maior tempo) e no teste de equilíbrio (maiores valores de COP PATH, COP VEL e área da Elipse, ou seja, maior oscilação do centro de pressão e em maior velocidade, portanto pior equilíbrio), tanto na situação de olhos abertos como fechados.

Já com relação ao grupo etário, as diferenças observadas foram apenas relacionadas à agilidade, o que demonstra que o tempo de dependência parece ter mais influência no equilíbrio corporal do que o avançar da idade. Particularmente no que se refere à agilidade, a perda de desempenho ao longo do envelhecimento, agravada pelos efeitos deletérios do consumo crônico de substâncias psicoativas, pode ser um fator que prejudique a realização de diversas tarefas do cotidiano de forma autônoma, tais como desviar de pessoas ou obstáculos e andar apressadamente com mudanças de direção. ${ }^{24}$

Nas análises correlacionais, tanto a idade quanto o tempo de uso mostraram relações significativas com o equilíbrio, evidenciando que quanto mais velho o indivíduo e quanto maior o tempo de uso de substâncias psicoativas, piores os resultados do teste na pla- 
taforma. No entanto, a variável COP Vel (variação do deslocamento do centro de pressão através do tempo) mostrou relação significativa apenas com o tempo de uso. Abrhamová \& Hlavacka ${ }^{25}$, avaliando o equilíbrio corporal de 81 indivíduos saudáveis divididos em três grupos etários (20-40, 40-60 e 60-82 anos), verificaram que a influência significativa da idade nas alterações dos parâmetros do centro de pressão (COP) ocorreu apenas a partir dos 60 anos. A este respeito, nenhum dos participantes do estudo era idoso, visto que a idade máxima observada na amostra foi de 56 anos. Assim, especula-se que, embora a idade seja um fator que influencie de forma independente o equilíbrio corporal e os dados sobre o tempo de uso devam ser interpretados com cautela, parece haver uma influência do tempo de consumo da substância psicoativa que ocorre mesmo em idades mais jovens. Neste sentido, destaca-se que os piores resultados observados no teste de equilíbrio corporal na amostra foram exibidos por um homem de 38 anos que era dependente de álcool há 15 anos.

Estudos afirmam que muitas drogas, incluindo o álcool, causam a tontura como efeito colateral, influenciando negativamente as habilidades motoras, abrangendo tarefas com tempo de reação simples, habilidades de coordenação e equilíbrio corporal ${ }^{6,7,26}$. Além disso, a dependência de álcool e drogas pode causar envelhecimento prematuro das funções neuropsicológicas e possivelmente do cérebro.

Brutto et al. ${ }^{9}$, em um estudo epidemiológico sobre a atrofia cerebelar induzida pelo alcoolismo, verificaram que a condição era presente em praticamente todos os casos, de maneira mais evidente naqueles com maior tempo de dependência e que consumiam quantidades maiores de álcool. Ainda Fein, Smith e Greenstein ${ }^{27}$, analisando indivíduos com alcoolismo simples ou combinado com a dependência de outras drogas, verificaram que o segundo grupo apresentou prejuízos significativos no equilíbrio corporal e na marcha quando comparados a indivíduos não dependentes. Segundo os autores, o consumo a longo prazo de álcool e outras drogas induz danos ao cerebelo, além de degradar a substância branca e comprometer a integridade do corpo caloso, ambas regiões relacionadas ao controle e estabilidade da postura corporal.

Embora não existam dados normativos para os parâmetros do centro de pressão em avaliações do equilíbrio corporal, alguns estudos realizados com indivíduos considerados saudáveis podem oferecer subsídios para a comparação com os dados obtidos no presente estudo.
Ghiringhelli \& Ganança ${ }^{28}$, ao analisarem 50 adultos de 18 a 25 anos ( 25 homens e 25 mulheres) com exames vestibulares e audiométricos normais, observaram área de Elipe e COP Vel, respectivamente, de $187,0+1,0 \mathrm{~mm}^{2}$ e $7,5+4,3 \mathrm{~mm} / \mathrm{s}$, valores estes muito próximos aos obtidos no presente estudo, à exceção dos indivíduos com mais de 10 anos de dependência química, que neste caso apresentaram valores de área da Elipse e COP Vel maiores $\left(213,3+142,9 \mathrm{~mm}^{2}\right.$ e 9,1 +6,3 $\mathrm{mm} / \mathrm{s}$, respectivamente). Da mesma forma que no presente estudo, os autores não verificaram diferenças nas variáveis entre os sexos.

Ainda Carvalho et al.29, analisando mulheres jovens $(21,0+2,0$ anos $)$ e idosas $(68,0+5,0$ anos), verificaram área de Elipse de $110 \mathrm{~mm}^{2}$ para o primeiro grupo e de $120 \mathrm{~mm}^{2}$ para o segundo, sem diferença significativa. Estes valores são muito inferiores aos encontrados no presente estudo com indivíduos com dependência química, talvez pelo fato de todas as mulheres pesquisadas pelos autores serem saudáveis e fisicamente ativas.

Assim, verifica-se que os efeitos do uso abusivo de álcool e outras drogas por um longo período pode afetar negativamente o equilíbrio corporal. Dependentes crônicos apresentam muitas vezes dificuldades em testes simples de equilíbrio e também quando caminham, manifestando uma marcha atáxica com base ampla ${ }^{5,7}$. Um leve esforço para estes indivíduos com lesão cerebelar possivelmente pode levá-los a ficar sem equilíbrio, mostrando sua falta de mecanismos para correção ${ }^{7}$.

Os prejuízos ao equilíbrio corporal e a outras capacidades coordenativas podem ser transitórios ou permanentes em indivíduos dependentes químicos, dependendo do tempo de uso e das características da substância, porém, ainda que algumas sequelas persistam, é possível que programas de intervenção com atividades físicas direcionadas amenizem tais sequelas, garantindo melhor controle corporal e autonomia a estes indivíduos ${ }^{5}$.

Algumas limitações devem ser mencionadas no presente estudo, tais como o recorte transversal, a ausência de um grupo controle (indivíduos sem dependência química), a baixa participação de pacientes do sexo feminino e a considerável variabilidade no tempo de dependência química. Ainda assim, é possível destacar que os dados aqui levantados podem oferecer subsídios relevantes para profissionais que atuam em serviços de saúde para esta população, de modo a ressaltar a importância de programas de intervenção direcionados à melhora da aptidão física destes indivíduos, favorecendo seu processo de recuperação e o risco de comorbidades associadas. 
De acordo com os resultados obtidos nas avaliações realizadas, pode-se concluir que a prevalência da amostra foi de homens com dependência múltipla de substâncias e com baixo nível de escolaridade. Ainda, aqueles dependentes de álcool foram em geral mais velhos e usavam a substância há mais tempo do que os demais. $\mathrm{O}$ avançar da idade mostrou para os indivíduos investigados influência negativa na agilidade, equilíbrio corporal e medida de cintura. Além disso, a dependência química afetou negativamente o comportamento da variável equilibrio corporal e os indivíduos com maior tempo de exposição à substância foram os mais prejudicados.

O presente estudo apresentou algumas limitações, tais como o número amostral reduzido e selecionado por conveniência, além da ausência da avaliação de algumas variáveis da aptidão física relacionada à saúde. Ainda assim, os dados aqui levantados são de relevância e interesse para os profissionais de saúde que atuam pessoas com dependência química, especialmente pela escassez de dados disponíveis sobre a condição física desta população, podendo assim oferecer subsídios para ações que envolvam a implementação de programas de exercícios físicos como estratégia terapêutica.

O número crescente de pessoas em situação de dependência química no Brasil impõe a necessidade iminente de informações mais aprofundadas sobre seu estado de saúde, a fim de que profissionais de diferentes áreas possam realizar suas intervenções de maneira embasada e específica. Assim, destaca-se a importância de novos estudos com essa população, com intervenções envolvendo protocolos de exercícios físicos, análises de outras variáveis como a capacidade cardiorrespiratória e a força muscular, e ainda amostras maiores e mais homogêneas, de modo a obter dados fidedignos sobre as características e o comportamento das variáveis da aptidão física desses indivíduos.

\section{Conflito de interesses}

Os autores declaram não haver conflito de interesses.

\section{Financiamento}

CAPES - Coordenação de Aperfeiçoamento de Pessoal de Nível Superior.

\section{Contribuição dos autores}

Malagodi BM participou da concepção inicial do estudo, planejamento e execução da coleta de dados e redação do texto. Greguol M participou da análise dos dados, redação e revisão crítica do texto. Serassuelo Jr H participou da concepção inicial do estudo, planejamento da coleta de dados e revisão crítica do texto.

\section{Referências}

1. American Psychiatric Association. DSM-5: manual diagnóstico e estatístico de transtornos mentais. 5. ed. Porto Alegre: Artmed; 2014.

2. Kalivas PW, Volkow ND. The neural basis of addiction: a pathology of motivation and choice. Am J Psychiatry. 2005;162(8):1403-13.

3. Stenbacka M, Leifman A, Romelsjö A. Mortality and cause of death among 1705 illicit drug users: a 37 year follow up. Drug Alcohol Rev. 2010;29(1):21-7.

4. Linke SE, Ussher M. Exercise-based treatments for substance use disorders: evidence, theory, and practicality. Am J Drug Alcohol Abuse. 2015;41(1):7-15.

5. Dolezal BA, Chudzynski J, Storer T, Abrazado M, Penate J, Mooney L, et al. Eight weeks of exercise training improves fitness measures in methamphetamine-dependent individuals in residential treatment. J Addict Med. 2013;7(2):122-8.

6. Flemmen G, Wang E. Impaired aerobic endurance and muscular strength in substance use disorder patients: implications for health and premature death. Medicine. 2015;94(44):1-7.

7. Schmidt PM, Giordani AM, Rossi AG, Cóser PL. Avaliação do equilíbrio em alcoólicos. Braz J Otorhinolaryngol. 2010;76(2):148-55.

8. Moreira DA, Ganança MM, Caovilla HH. Posturografia estática em dependentes de drogas ilícitas e álcool. Braz J Otorhinolaryngol. 2012;78(5):97-103.

9. Brutto OHD, Mera RM, Sullivan LJ, Zambrano M, King NR. Population-based study of alcoholic cerebellar degeneration: The Atahualpa Project. J Neurol Sci. 2016;367:356-60.10.

10. Ahmad S, Rohrbaugh JW, Anokhin AP, Sirevaag EJ, Goebel JA. Effects of lifetime ethanol consumption on postural control: a computerized dynamic posturography study. J Vestib Res. 2002;12(1):53-64.

11. Folstein MF Folstein SE, McHugh PR. Mini Mental state. J Psychiat Res. 1975;12:189-98.

12. American Psychiatric Association. Código Internacional de Doenças. Washington, DC, USA: APA, 2013. Disponível em: <http://www.cid10.com.br/>. Acesso em: 20 jan. 2018.

13. World Health Organization. Global Database on Body Mass Index. Geneva; 2002 [citado 2017 mai 22]. Disponível em: http://www.who.int/bmi/index. jsp?introPage=intro_3.html.

14. Donath L, Roth R, Zahner L, Faude O. Testing single and double limb standing balance performance: comparison of COP path length evaluation between two devices. Gait Posture. 2012;36(3);439-43.

15. Wells KF, Dillon EK. The sit and reach - a test of back and leg flexibility. Res Q. 1952;23:115-8.

16. Pauole K, Madole K, Garhammer J, Rozenek R. Reliability and validity of the t-test as a measure of ability, leg power and leg speed in college-aged men and women. J Strength Cond Res. 2000;14(4):443-50.

17. Johns A. Psychiatric Effects of Cannabis. Br J Psychiatry. 2001;178:116-22.

18. Secretaria Nacional de Políticas sobre Drogas (SENAD) [homepage na internet]. Pesquisas sobre o consumo de drogas no Brasil [acesso em 01/10/2017]. Disponível em http://www.aberta.senad.gov.br/.

19. Organização Mundial da Saúde - World Health Organization (WHO) [homepage na internet]. Drug abuse [acesso em 14/06/2017]. Disponível em http://www.who.int/. 
20. Willhelm FF, Escobar M, Perry IDS. Alterações na composição corporal e em parâmetros antropométricos de dependentes de crack internados em unidade de adição. J Bras Psiquiatr. 2013;62(3):183-90.

21. Lipowski M, Szulc M, Buliński L. Physical activity among other health-related behaviors in treatment of alcoholism. J Sports Med Phys Fitness. 2015;55(3);231-40.

22. Appelqvist-Schmidlechner K, Vaara J, Mäkinen J, Vasankari T, Kyröläinen H. Relations between leisure time physical activity, physical fitness and mental health among young adult males. Eur Psychiatry. 2017;41:S179.

23. Galper DI, Trivedi MH, Barlow CE, Dunn AL, Kampert JB. Inverse association between physical inactivity and mental health in men and women. Med Sci Sports Exerc. 2006;38(1):173-8.

24. Zago AS, Gobbi S. Valores normativos da aptidão funcional de mulheres de 60 a 70 anos. Rev Bras Ciên Mov. 2008;11(2):77-86.

25. Abrahamova D, Hlavacka F. Age-related changes of human balance during quiet stance. Physiol Res. 2008;57(6):957-64.
26. Fein G, Greenstein D. Gait and Balance Deficits in Chronic Alcoholics: No Improvement from 10 Weeks Through 1 Year Abstinence. Alcohol Clin Exp Res. 2013;37(1):86-95.

27. Fein G, Smith S, Greenstein D. Gait and Balance in Treatment - Nave Active Alcoholics with and without a Lifetime Drug Codependence. Alcohol Clin Exp Res. 2012;36(9):1550-62.

28. Ghiringhelli R, Ganança CF. Posturografia com estímulos de realidade virtual em adultos jovens sem alterações do equilíbrio corporal. Rev Soc Bras Fonoaudiol. 2011;23(3):264-70.

29. Carvalho CE, Silva RA, Gil AW, Oliveira MR, Nascimento JA, Pires-Oliveira DAA. Relationship between foot posture measurements and force platform parameters during two balance tasks in older and younger subjects. J Phys Ther Sci. 2015;27(3):705-10.

Recebido: 20/10/2017

Aprovado: 06/02/2018

\section{Como citar este artigo:}

Malagodi BM, Greguol M, Serassuelo Junior H. Análise do equilibrio corporal e aptidão física de indivíduos em tratamento para dependência química. Rev Bras Ativ Fís Saúde. 2018;23:e0012. DOI: 10.12820/rbafs.23e0012 